\title{
Tomšič Čerkez, B. and Zupančič, D. (2011). Play Space [Prostor igre]. Ljubljana: University of Ljubljana, Faculty of Education and Faculty of Architecture. 164 p., ISBN 978-961-253-053-2.
}

Reviewed by Borut Juvanec

Children are our future, play is their work. Work strengthens children and develops their capacities, skills and thinking. So it matters how a child plays. Above all, it matters where s/he plays, how s/he grasps the environment, since play connected with a space encourages discoveries. In a restricted, dark, badly organised space that is poorly articulated, a child cannot develop a feeling for clear thinking, for wide recognition, for relations between people, for freedom of thought.

So the space in which a child lives, and in which s/he works, is very important.

Not a lot of books have been written about play, especially not in the field of architecture, the design of space. The present publication is not just a handbook on the theme of play and planning playgrounds; as well as reviewing existing solutions in the world, it analyses them, considers them and proposes further solutions.

The authors have systematically, critically and fully developed the subject of play and its impact on architectural composition in space: at first entirely theoretically, with examples and possibilities given.

Space is always limited by actual, physical and apparent, psychological elements. Limitation is a particularly important element of children's education; it can influence them negatively or help them to develop thought processes in connection with space. A well-designed space must enable play, encouraging and developing it. In this context, two professions are important: pedagogy and architecture. By providing possibilities, architecture develops the child's thinking, so that the child actively intervenes in the architecture; so that in the given possibilities the child invents something new, something that is actually original but has been unobtrusively provided by the architect. Pedagogy supplements its own intervention with elements of architecture, which thus simultaneously become limitation and encouragement.

The combination of the two sciences is much more than merely their sum.

For example, a fence is a restriction intended for safety, but sometimes also ensuring survival. The detail of a fence that is adapted to the hand is an 
important learning element for a child; the child studies it, understands it and finally grasps it as a functional detail that is useful for him/her. Then s/he no longer notices it - until it is no longer there. Then the child misses it: it is good, and s/he simply no longer remembers the bad. Nature is said to be cruel; so is a child, who links elements in an unbelievably imaginative way. This sense leads a child to simple solutions that are functional, comfortable and, in the end, beautiful. Such, too, is architecture, especially in wood. Wood is a warm, human material, which a child in particular knows how to appreciate if it is elementary, simple and functional. A child can thus be a test for design: if s/he likes a detail it is certainly good. The authors talk about the life cycle, part of which is certainly searching, recognising and understanding space.

Exterior and interior spaces, which the authors distinguish in accordance with external influences, are extremely important in this. In bad weather conditions, a child quickly turns to the inside; in good weather, s/he wants to go out. Good architecture enables this; moreover, through the apparent links between interior and exterior spaces it encourages observation. Otherwise, how is a child to understand the physical properties of water between liquid, steam and ice? Nature is the best teacher; it need only be allowed to show these properties. The authors demonstrate precisely this example, and in this they have an extraordinary advantage: multi-professionalism.

Their relation thus has even greater importance, as they write it as several experts: two architects, a pedagogue, a painter and an economist. The authors of the book are both simultaneously professionally and scientifically proficient and personally committed. What is relevant here is that they are not just speaking on the basis of foreign experiences, but above all on the basis of their own experiences; and they did not have children 'sometime in the last century'; they have them here and now. This is apparent throughout the book and in every detail. They are writing with love, although their scientific approach sometimes restricts this.

The basic findings of the content are:

- $\quad$ Play is a way of growing up in a space in which relations among individuals are established and bonds between individuals in the space are reinforced. Play enables the discovery of a child's own identity and his/her own capacities in relation to himself/herself, to others, to the community, to various social groups and, of course, to the space. The space of play defines an individual and his/her sense of survival. Good play is play that enables reflection and offers an individual his/her own and common active expression.

- The contents of the book raise the question of planning spaces in 
which the language of play begins to find full expression (boldness, investigation, laughter, joy, expressing ideas and thoughts, creativity).

- Education is thus present at every moment: all activities are subordinate to education, deliberately unconsciously - for the child, for the teacher and for parents. This is precisely the result of the considered design of space that enables these activities, encouraging and developing them.

- The book discusses the elements that are the foundation for this: shape, colour, order of elements, details and the whole, beginning and end, entrance with or without exit. These are elements that are only apparently merely technical; in the hands of real experts, they become tools for education, functioning unconsciously and effortlessly. Moreover, in the right environment work is fun. If we look at a child who is creating, s/he is serious; above all, this is work for him/her. It is also important that a child evaluates work very strictly and appreciates it. For us, this is play; for the child, it is work. This needs to be understood.

- Nor should it be overlooked that children are not stupid, that they understand things about which adults only whisper in front of them. The environment, the space, is precisely the element that enables this.

The last complete monograph dealing with the sphere of play was written in the 1970s, more than 30 years ago. A great deal has changed since then; new needs and new possibilities have appeared. Together with needs, new possibilities for implementation are opened every day: the availability of materials, their quality, breadth of use, appearance and final finish and, not least, their economics. With ever greater purchasing power - regardless of oscillations and recessions - we must choose only the best for children, as a Scottish proverb says.

So the book Play Space is welcome and necessary for architects in planning, for pedagogues in bringing up groups, and for parents in bringing up individuals, as well as being useful in general for the child.

The book is divided into chapters such as Play, Space, Play Equipment and Play Space. At the end, as any scientific publication, it has the scientific appendices, here presented as References, Abstracts in Slovene, English, Castilian and German, an Index and a Biography of the two authors.

The chapter Play talks about the importance of play for children, about development, and about the senses that are bound to space, discussing perception and experience. These are the theoretical foundations, revealing questions while simultaneously pointing to answers.

The second chapter is entitled Space. Here the authors consider mankind in space, with particular stress on children in space intended for them and 
in space intended for adults. The authors continue by dealing with art and the design of space that is educational and laboratory, thus theoretical and practical. A playground is an especially important element, as an external space that is differentiated and yet simultaneously connected with the interior.

Play Equipment is a very important section. In this chapter, the authors consider the theory of design that enables, encourages and controls play. In particular, the idea of order and composition is distinguished, which enables a division of work, linkage, superstructure and encourages active and directed thinking. The importance of the design of equipment for play as a subject of the 'design of space' (= architecture) is actually the practical implementation of theoretical foundations. Materiality introduces a new concept of the choice of materials, the relation to the materials and the location, which rounds out the life cycle, both of the child and of the play equipment, for parents and teachers, for the organisers of education and for architects. An important contribution of this thinking is the importance of variety, which is expressed in play and in play equipment and is the fruit of both the architect and the teacher, taking into account the wide scope of the profession and science.

The monograph is sensibly planned, with a clear index including subheadings, while the chapters are separated by coloured pages, clearly indicating the organisation and content of the book. This clarity is reinforced by the presentation all of the scientific findings in graphic form.

The book is divided into chapters that end with 'conclusions', providing a kind of summary of the problem described. Since it is not possible to translate everything, these conclusions are translated into four languages, which thus provide a summary of the whole book in educational terms as the authors develop some details in the book itself. If these conclusions are combined into a unitary text, we obtain the content of the book in brief, as well as its emphases. Although perhaps not planned by the authors, the result is well considered, as the system by which they have developed the question of the design of a play space. As with space, they have arranged the chapters one after another so that they are logically connected and mutually interwoven. Thinking about space thus obtains a completely new meaning, clear and logical, through the composition of the elements of the book itself. Education, therefore, which has developed by itself. The best education, attested in the place itself.

Today, we must do everything within our power to enable a child to develop into a free individual with a clearly expressed personality; this is a condition for the socialisation of each individual and for the shaping of society as a whole. Society is a composition of individuals, and the strength of a society is that which is brought by its weakest link. 
It is precisely play that is an element that enables the development of the less talented, less developed and less comprehensive individual, to whom we must provide at least equal opportunities.

Design and graphics are elements that unconsciously bring aspects of education close to a child, enabling him/her to understand things that $s /$ he would not otherwise grasp.

Space is more important than we imagine.

And play has more importance than we ascribe to it.

And a child is the most important of all. If only the best is good enough for a child, this is also true for designing the environment in which s/he lives and develops.

I am particularly pleased that the Faculty of Pedagogy and the Faculty of Architecture are the co-publishers within the framework of the University of Ljubljana. I am an architect myself and, of course, I see space from my own point of view and with my own limited knowledge. Architects are often reproached for being one-sided, often justifiably. On this occasion, this one-sidedness has been surpassed, despite the fact that both of the authors are architects, the theme has been scientifically treated yet reads as a story. The educational element, about which the authors continually speak, is thus applied first to themselves and only then to others. This is fair, and it is successfully implemented.

It should not be overlooked that the authors are not talking about work in the book, they are working. They have worked, in fact. The illustrative material is carefully chosen and supplemented with diagrams, something that is missing in some books on similar themes (in architecture as well as in pedagogy). The sketch is not an end in itself - it does not have artistic ambitions and does not attract attention - it simply supplements the content and explains the functioning or the impact of design in space.

The excellent, hand-drawn line expresses the content of the tale and the character of a man who knows what he wants and knows what needs to be said. A drawing says more than a thousand words.

This does not just apply to both of the authors; the carefully selected pictures by youngsters supplement the thinking of adults and the pictures from work testify to the enthusiasm, desires and aspirations of the young generation, which wants to actively intervene in its environment, an environment that adults tailor for it.

This, though, is already the content of the book, which talks about architecture and about education but says much more.

This excellent book opens up some problems that to date have not been recognised, have not been articulated; it explains, describes and proposes 
solutions for tomorrow. Perhaps the present book in this field will be followed by a similar work from the psychological point of view, from the linguistic, historical or artistic perspective, recording the characteristics of the individual, special groups, local characteristics or the culture of society, space, time, culture.

That will already be a new book; it cannot surpass Play Space. 